EPJ Web of Conferences 73, 01010 (2014)

DOI: $10.1051 /$ epjconf/20147301010

(C) Owned by the authors, published by EDP Sciences, 2014

\title{
Results and perspective on TMDs and GPDs at COMPASS
}

\author{
Nicole d'Hose ${ }^{\mathrm{a}}$ on behalf of the COMPASS Collaboration \\ CEA Saclay, Irfu/SPhN, 91191 Gif-sur-Yvette, France
}

\begin{abstract}
COMPASS is a fixed target experiment at the CERN SPS dedicated to the study of the nucleon structure in the framework of the most complete descriptions based on transverse momentum dependent parton distribution functions (TMDs) and generalised parton distributions (GPDs). TMDs have been accessed so far with semi-inclusive deep inelastic reactions using a $160 \mathrm{GeV}$ muon beam off transversely polarized targets and they will be further investigated with Drell-Yan reactions using a $190 \mathrm{GeV}$ pion beam off a transversely polarized proton target. GPDs will be studied with deeply virtual Compton scattering and hard exclusive meson production using muon beams. A selection of the results as well as the projections for the future program are discussed.
\end{abstract}

\section{Introduction}

The description of the partonic structure of the nucleon is one of the central problems of hadronic physics. The most general information on the partonic structure of hadrons is contained in the generalised parton correlation functions (GPCFs) [1] which parameterise the fully unintegrated, off diagonal quark-quark correlators for a given hadron. These functions have a direct connection to the so-called Wigner distributions - the quantum mechanical analogues of classical phase space distributions - of the hadron-parton system. The GPCFs are "mother distributions" of the generalised parton distributions (GPDs) and the transverse momentum dependent parton distributions (TMD PDFs or TMDs), which can be considered as different projections or limiting cases of GPCFs. These two types of parton distributions, GPDs and TMDs, provide a 3-dimensional picture of the nucleon, either in a mixed position-momentum representation for the former or in a pure momentum space for the latter. Moreover they contain important information on the orbital motion of partons inside the nucleon. While GPDs appear in the QCD description of hard exclusive reactions such as deeply virtual Compton scattering (DVCS) or hard exclusive meson production, TMDs can be measured in the semi-inclusive deep inelastic scattering (SIDIS) or the Drell-Yan process.

COMPASS is a fixed target experiment at the CERN SPS taking data since 2002. Transverse spin and transverse momentum effects are studied with SIDIS data collected using a $160 \mathrm{GeV}$ muon beam and transversely polarised proton $\left(\mathrm{NH}_{3}\right)$ and deuteron $\left({ }^{6} \mathrm{LiD}\right)$ targets. The spectrometer [2] comprises a variety of tracking detectors and allows to detect charged tracks in a broad momentum and angular range. Calorimeters, muon filters and a gas RICH detector are available for particle identification.

\footnotetext{
a e-mail: nicole.dhose@cea.fr
}

This is an Open Access article distributed under the terms of the Creative Commons Attribution License 4.0, which permits unrestricted use, distribution, and reproduction in any medium, provided the original work is properly cited. 

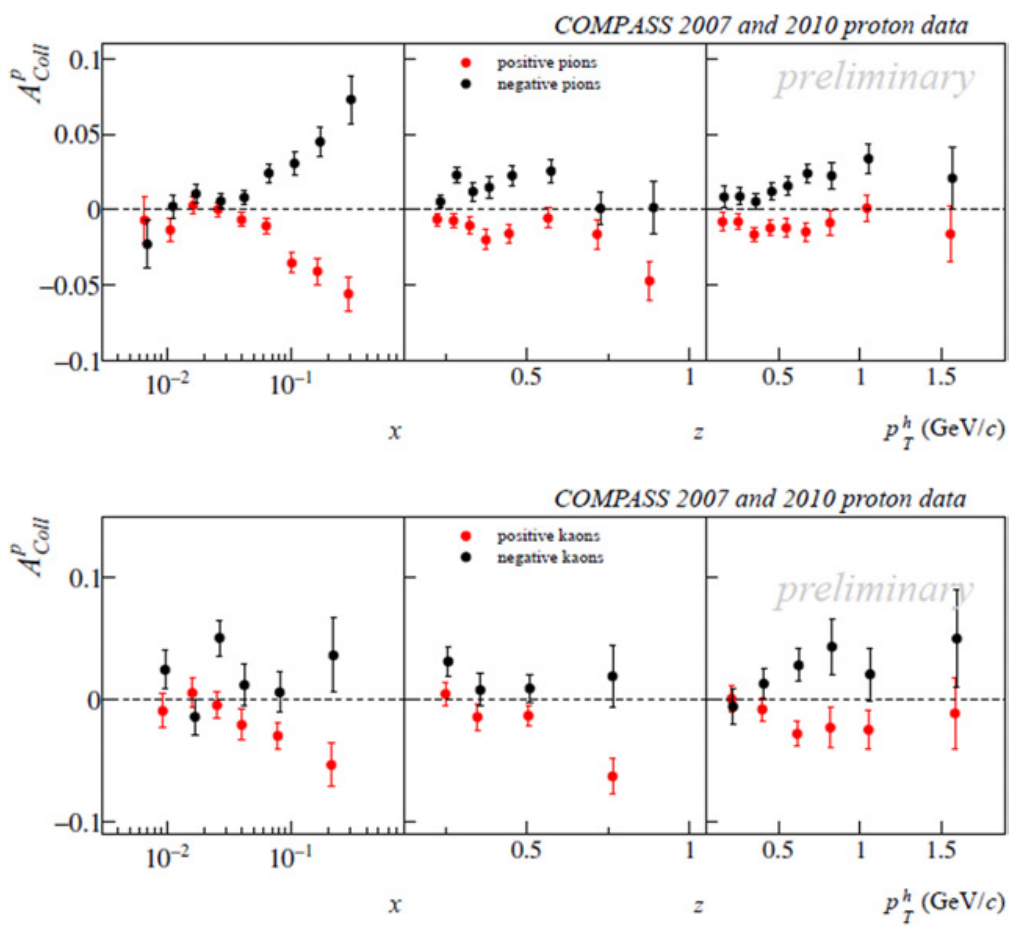

Figure 1. The Collins asymmetries for pions (top) and kaons (bottom) measured at COMPASS, as a function of $x$, $z$, and $p_{T}^{h}$.

The future COMPASS program [3] starting in 2014 after the accelerator shutdown focuses on TMDs and GPDs. TMDs will be further investigated with a polarised Drell-Yan experiment in 2014-15 using a $190 \mathrm{GeV}$ pion beam off a transversely polarized proton target. GPDs will be studied in 2016-17 with deeply virtual Compton scattering and hard exclusive meson production using muon beams and a liquid hydrogen target surrounding by a recoil detector.

\section{TMD studies with SIDIS experiments}

In (un)polarised SIDIS, where a hadron is detected in the final state, TMD PDFs can be obtained by the study of the azimuthal asymmetries. For a transversely polarised target there are 4 leading twist asymmetries which are sensitive to the convolution of a TMD PDF with the corresponding fragmentation function $(\mathrm{FF})$ :

- $A_{U T}^{\sin \left(\phi_{h}+\phi_{S}-\pi\right)} \propto h_{1} \otimes H_{1}^{\perp}$ with $h_{1}$ the transversity PDF and $H_{1}^{\perp}$ the Collins FF (Collins asymmetry);

- $A_{U T, T}^{\sin \left(\phi_{h}-\phi_{S}\right)} \propto f_{1 T}^{\perp} \otimes D_{1}$ with $f_{1 T}^{\perp}$ the Sivers PDF and $D_{1}$ the unpolarised FF (Sivers asymmetry);

- $A_{U T}^{\sin \left(3 \phi_{h}-\phi_{S}\right)} \propto h_{1 T}^{\perp} \otimes H_{1}^{\perp}$ with $h_{1 T}^{\perp}$ the pretzelosity PDF and $H_{1}^{\perp}$ the Collins FF;

- $A_{U T}^{\cos \left(\phi_{h}-\phi_{S}\right)} \propto g_{1 T} \otimes D_{1}$ with $g_{1 T}$ the worm-gear PDF and $D_{1}$ the unpolarised FF.

The first and second subscripts indicate the beam and target polarizations (U: unpolarised, L: longitudinal and $\mathrm{T}$ : transverse), the third subscript the virtual photon polarization and the superscript the azimuthal modulation. With an unpolarised target the Boer Mulders PDF can be reached:

- $A_{U U}^{\cos \left(2 \phi_{h}\right)} \propto h_{1}^{\perp} \otimes H_{1}^{\perp}$ with $h_{1}^{\perp}$ the Boer-Mulders PDF and $H_{1}^{\perp}$ the Collins FF. 

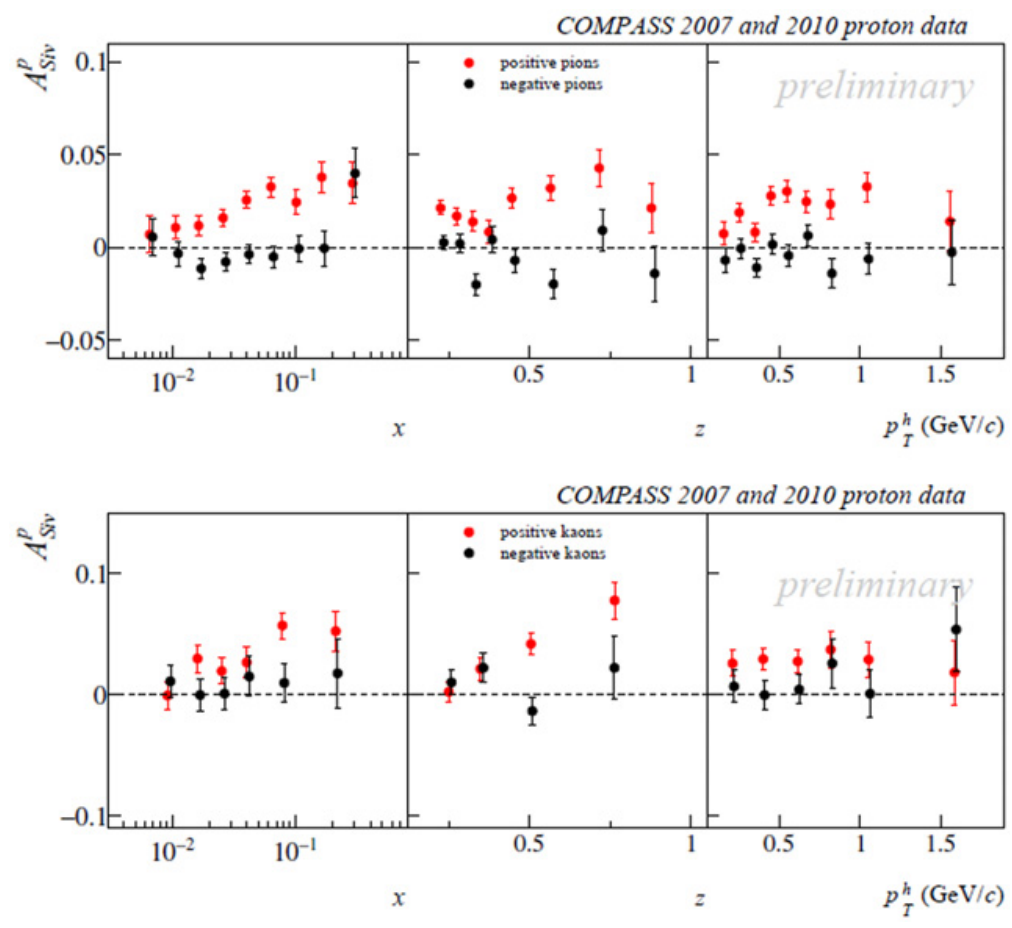

Figure 2. The Sivers asymmetries for pions (top) and kaons (bottom) measured at COMPASS, as a function of $x$, $z$, and $p_{T}^{h}$.

Note that $h_{1}, h_{1 T}^{\perp}, h_{1}^{\perp}$ are chiral-odd and require a chiral-odd FF given by $H_{1}^{\perp}$, the Collins FF, describing correlation between the quark spin and the transverse momentum of the produced hadron. Note that the Sivers and Boer-Mulders PDFs are T-odd.

The Collins asymmetries for charged pions and kaons from all the collected proton data at COMPASS are shown in Fig. 1, as a function of the Bjorken variable $x$, the relative energy of the hadron $z$, and the hadron transverse momentum $p_{T}^{h}$. The pion asymmetries are very similar to the published unidentified hadron asymmetries [4]: at small $x$ the asymmetries are compatible with zero, while in the valence region, they show an increasing signal, of opposite sign for $\pi^{+}$and $\pi^{-}$. The result for charged kaons, even if affected by large error bars, show a similar trend. In the large $x$ region, these results are in good agreement with those of HERMES [5], a non obvious result due to the different kinematic range covered: in the last $x$ bins, the COMPASS $Q^{2}$ is larger by a factor 2-3 wirh respect to the HERMES one.

All the asymmetries measured by COMPASS on deuteron $[6,7]$ are found to be compatible with zero, also for kaons. This result is interpreted as a cancellation between the $\mathrm{u}$ and $\mathrm{d}$ quark contribution in a isoscalar target.

A combined fit [8] of the SIDIS data from HERMES and COMPASS together with the $e^{+} e^{-}$BELLE data [9] allows the simultaneous extraction of $h_{1}$ for the $\mathrm{u}$ and d quarks with the interesting byproduct that the unfavoured Collins FF is opposite to and as large as the favoured one.

The Sivers asymmetries for charged pions and kaons from all the collected proton data at COMPASS are shown in Fig. 2. Also in this case the pion asymmetries are very similar to the published unidentified hadron asymmetries [4]. The asymmetries for negative pions and kaons are compatible with zero, while for positive pions and kaons there is an evidence for a positive signal extending overall the measured $x$ region, and increasing with $z$. Very intriguing is the fact that the $K^{+}$signal is larger than the 
$\pi^{+}$one, hinting for a possible not negligible role of the sea quarks. At variance with the Collins effect, at large $x$ the COMPASS asymmetries are smaller by a factor of two wirh respect to the HERMES ones[10], giving insights of the $Q^{2}$ evolution of the Sivers functions.

All the asymmetries measured by COMPASS on deuteron $[6,7]$ are found to be compatible with zero, also for kaons. This result is interpreted as a cancellation between the $\mathrm{u}$ and $\mathrm{d}$ quark contribution in a isoscalar target.

Other six transverse spin asymmetries have been measured at COMPASS in SIDIS. The $A_{U T}^{\sin \left(3 \phi_{h}-\phi_{S}\right)}$ asymmetry, giving access to the prezelosity PDF, has been found compatible with zero both on deuteron and proton targets. Regarding the $A_{U T}^{\cos \left(\phi_{h}-\phi_{S}\right)}$ asymmetries, giving access to the worm-gear PDF, while the results on the deuteron target are compatible with zero, the proton results show a small positive signal increasing with $x$. A similar trend has been observed in HERMES preliminary results and in the JLab E06-00 results on neutron target.

Unpolarised asymmetries have also been measured at COMPASS in SIDIS using an unpolarised isoscalar target ( $\left.{ }^{6} \mathrm{LiD}\right)$. Among the three independent modulations, the $A_{U U}^{\cos \left(2 \phi_{h}\right)}$ one gives access to the leading twist Boer-Mulders PDF. This asymmetry presents strong dependence with the kinematical variables: the signal is large at small $x$ and decreasing with $x$ and with an intriguing dependence in $p_{T}^{h}$. Thanks to the large cumulated statistics a multi-dimensional analysis has also been performed.

The SIDIS measurements on a proton using a liquid hydrogen target will be pursued in 2016-17 in parallel with DVCS and hard exclusive meson production for GPD studies.

\section{TMD studies with Drell-Yan experiment}

The second approach to study TMDs in COMPASS is via the Drell-Yan (DY) process using a $190 \mathrm{GeV}$ negative pion beam impinging on a transversely polarised proton target $\left(\mathrm{NH}_{3}\right)$. The advantage of DY process is that FF are not involved. However, this has to be paid by a convolution of two PDFs. The DY cross-section is given by $\sigma^{D Y} \propto f_{\bar{u} \mid \pi^{-}} \otimes f_{u \mid p}^{\prime}$ where $f$ and $f^{\prime}$ are generic PDFs and where the $\pi^{-} p \rightarrow \mu^{+} \mu^{-} X$ reaction is dominated by the $\bar{u} u$ quark annihilation. Polarised DY experiments can study TMDs like the Sivers and the Boer-Mulders PDFs. Theory predicts that these T-odd TMDs obey a restricted universality and change sign when observed in SIDIS and DY:

$$
\left.f_{1 T}^{\perp}\right|_{D Y}=-\left.f_{1 T}^{\perp}\right|_{D I S} \quad \text { and }\left.\quad h_{1}^{\perp}\right|_{D Y}=-\left.h_{1}^{\perp}\right|_{D I S} .
$$

This sign change is due to switching from final-state interaction in SIDIS to initial-state interaction in DY [11]. Given the clear signals measured at COMPASS in SIDIS, the direct comparison of these TMD symmetries obtained from SIDIS and DY in the same kinematic domain is of great interest. Many plans for polarised DY measurements exists also at various laboratories, e.g. Fermilab, RHIC, JPARC, GSI and NICA (JINR).

In order to minimize the contribution from the $J / \psi$, charmed meson decays and the combinatorial background (secondary pions decay into muon and neutrino), the experiment will focus on high mass dimuon region $4 \mathrm{GeV}<M_{\mu \mu}<9 \mathrm{GeV}$. The azimuthal asymmetries depend on $\phi_{S}$, the azimuthal angle of the target spin wirh respect to the transverse momentum of the virtual photon in the target rest frame, on $\phi$, the azimuthal angle between the incoming hadron and outgoing lepton plane in the Collins-Soper frame, on $\theta$, the polar angle of the lepton pair, and on the Feynman variable $x_{F}=x_{\pi}-x_{p}$. Here $x_{\pi}$ and $x_{p}$ are the momentum fractions carried by the involved quarks in the pion and proton, respectively. The Sivers related asymmetry $A_{T}^{\sin \phi_{S}}$ is compared to predictions in Fig. 3 (left). The first ever test of the sign change of the T-odd Sivers PDF when accessing it from DY or from SIDIS will be performed, the absolute size of the effects will be measured as well. The Boer-Mulders related asymmetry $A_{U}^{\cos 2 \phi}$, shown in Fig. 3 (right) will also be determined with high precision. 

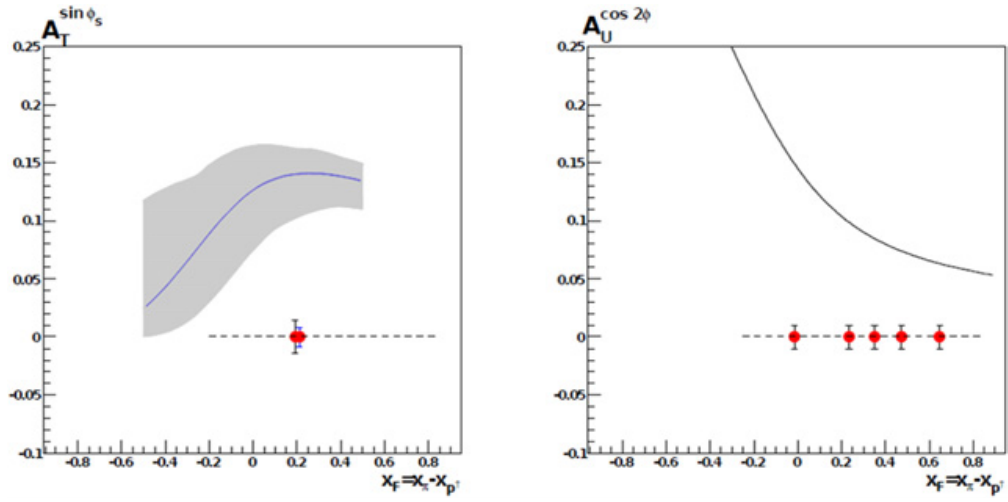

Figure 3. Projections for the $A_{T}^{\sin \phi_{S}}$ (Sivers) and $A_{U}^{\cos 2 \phi}$ (Boer-Mulders) asymmetries for $4 \mathrm{GeV}<M_{\mu \mu}<9 \mathrm{GeV}$. The shaded grey band and the central line correspond to a calculation based on a TMD PDF fit to data [12].

As the measurement is statistics limited, optimising luminosity is mandatory. A hadron absorber downstream of the target reduces radiation and detector occupancy problems. A rearrangement of the target area with the absorber, a new di-muon trigger and an additional vertex detector providing a clear separation of the two target cells are being prepared for the data taking starting in 2014.

\section{GPD studies with exclusive reactions}

The GPDs are universal distributions which embody both, form factors observed in elastic scattering and parton distribution functions measured in deep inelastic scattering. So GPDs can be considered as a momentum-dissected form factors providing information on the transverse localisation of a parton as a function of $x$, the fraction it carries of the nucleon's longitudinal momentum. Note that for exclusive reactions the fraction $x$ is not the Bjorken scaling variable, which will be noted $x_{B j}$ in the following. DVCS and hard exclusive meson production are the most promising ways to experimentally constrain the GPDs. COMPASS with the availability of $160 \mathrm{GeV}$ muon beams will explored the uncharted $x_{B j}$ domain between the HERA collider experiments H1 and ZEUS and the fixed-target experiments as HERMES and JLab (presently the only active laboratory with its upgrade at $12 \mathrm{GeV}$ ).

The existing COMPASS set up will be equipped with a $2.5 \mathrm{~m}$ long liquid hydrogen target surrounding by a recoil detector, CAMERA. This detector mandatory to assure the exclusivity of the observed reactions, is already built and has been tested during the one month pilot run in 2012. A third calorimeter, ECAL0, placed just downstream of the target, was also installed and will be still enlarged for the future data taking. This increases significantly the acceptance of the DVCS photon detection.

The DVCS process interferes with the Bethe-Heitler $(\mathrm{BH})$ process due to identical final states. The cross-section for hard exclusive muoproduction of a single photon off an unpolarised proton can be written as:

$$
\mathrm{d} \sigma^{(\mu p \rightarrow \mu p \gamma)}=\mathrm{d} \sigma^{B H}+\mathrm{d} \sigma_{0}^{D V C S}+P_{\mu} \mathrm{d} \Delta \sigma^{D V C S}+e_{\mu} \operatorname{Re} I+e_{\mu} P_{\mu} \operatorname{Im} I,
$$

where $I$ denotes the DVCS-BH interference term. An important feature is that the BH contribution dominates at small $x_{B j}$ and thus provides an excellent reference yield to monitor the detector acceptance and the luminosity measurement. From the previous equation one can build the sum $\mathcal{S}$ and difference $\mathcal{D}$ of the cross-section for simultaneous change of lepton charge $e_{\mu}$ and polarisation $P_{\mu}$ of the incoming 

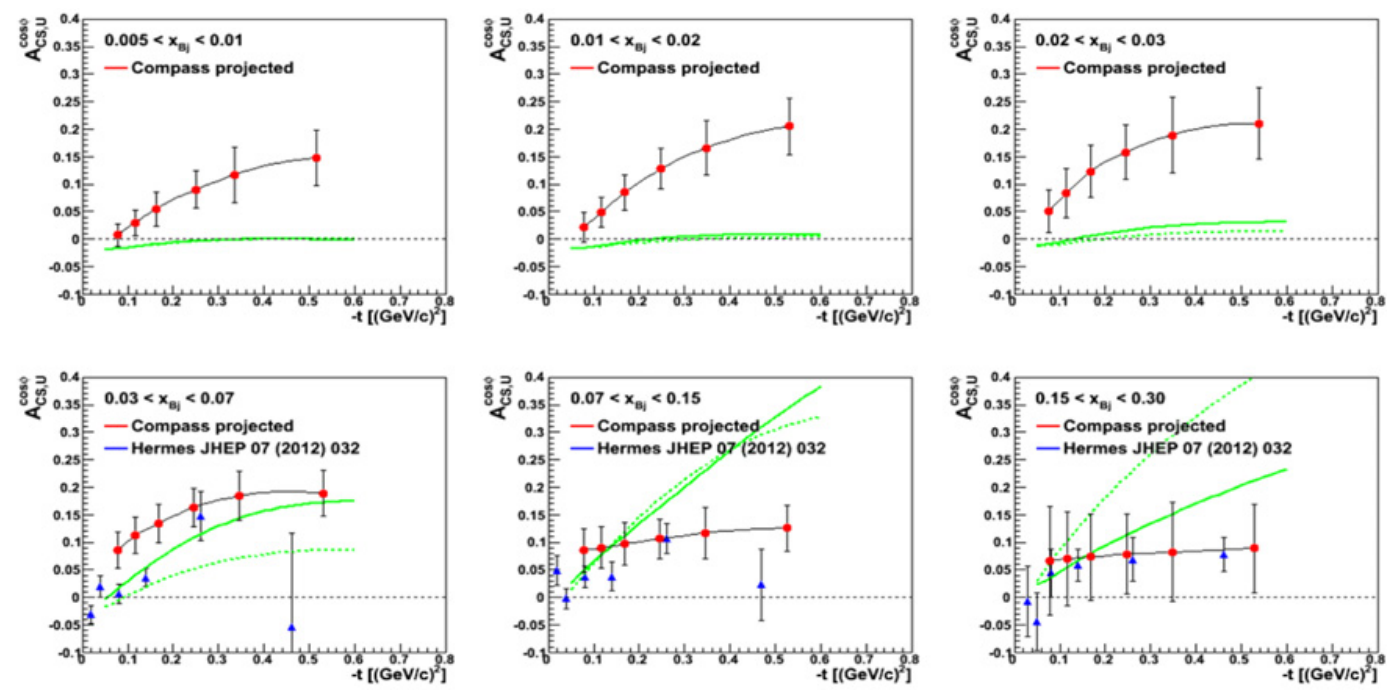

Figure 4. Projected statistical accuracy for the amplitude of the $\cos \phi$ modulation of the beam charge and spin asymmetry using the 3 calorimeters. Projections (red points) are calculated using the reggeised variant of the VGG model [14]. The green curves show the latest predictions [15] based on the first fit on world data including JLab Hall A (solid line) or not (dotted line). The blue triangles at large $x_{B j}$ show HERMES results [16].

lepton beam $(+$ to - and $\leftarrow$ to $\rightarrow$ )

$$
\begin{gathered}
\mathcal{D} \equiv \mathrm{d} \sigma^{\leftarrow}-\mathrm{d} \sigma^{-}=2\left[\mathrm{~d} \Delta \sigma^{D V C S}+\operatorname{Re} I\right] \stackrel{\text { L.O. }}{\longrightarrow}\left(c_{0}^{I}+c_{1}^{I} \cos \phi\right) \\
\mathcal{S} \equiv \mathrm{d} \sigma^{+}+\mathrm{d} \sigma^{-}=2\left[\mathrm{~d} \sigma^{B H}+\mathrm{d} \sigma_{0}^{D V C S}+\operatorname{Im} I\right] \stackrel{\text { I.O. }}{\longrightarrow} 2 \mathrm{~d} \sigma^{B H}+c_{0}^{D V C S}+s_{1}^{I} \sin \phi .
\end{gathered}
$$

The muon beam used at COMPASS has exactly this behaviour that negative muons have opposite polarisation than positive muons. Using a proton target in the COMPASS kinematics the coefficient $c_{i}^{j}$ or $s_{i}^{j}$ are related to the dominant Compton form factor (CFF) $\mathcal{H}$ which is a convolution of the GPD $H$ with a kernel describing the photon - quark interaction [13].

Upon integration over the azimuthal angle $\phi$ the interference contribution to $\mathcal{S}$ vanishes and after subtraction of the BH contribution one obtains the pure DVCS cross-section. Its depends on the squared momentum transfer $t$ from the initial to the final nucleon. If the behaviour $\mathrm{d} \sigma_{0}^{D V C S} / \mathrm{d} t \propto$ $\exp \left(-B\left(x_{B j}\right)|t|\right)$ is confirmed with the data, and using the relation $<r_{\perp}^{2}\left(x_{B j}\right)>\approx 2 B\left(x_{B j}\right)$ valid at small $x_{B j}$, one can extract the transverse distance $r_{\perp}$ measured between the struck quark and the centre of mass of the spectator system. Thus, independently of any GPD parameterisation, one obtains a measurement of the transverse nucleon size.

The $\phi$ dependences of the difference $\mathcal{D}$, the sum $\mathcal{S}$ and the asymmetry $\mathcal{A}=\mathcal{D} / \mathcal{S}$ allow for the extraction of quantities related to the CFF $\mathcal{H}$ and thus constrain the GPD $H$. The statistics expected in two years of data taking will permit to investigate 6 bins in $x_{B j}$ ranging from 0.005 to 0.3 , 6 bins in $t$ ranging from 0.06 to $0.7 \mathrm{GeV}^{2}$ and 4 bins in $Q^{2}$ from 1 to $16 \mathrm{GeV}^{2}$. Figure 4 shows the $\cos \phi$ modulation of the asymmetry, integrated over $Q^{2}$ as a function of $t$ in 6 domains in $x_{B j}$. This modulation is related to the real part of the CFF $\mathcal{H}$. Note that it exists a dispersion relation between the real and imaginary parts of the CFF with a subtraction D-term. The D-term, related to the confinement of partons in the nucleon, is not well constrained by the present data. The real part of the CFF $\mathcal{H}$ was found positive at HERA and negative at HERMES. The kinematic domain of COMPASS, in particular the region $0.005<x_{B j}<0.03$ 
(see the 3 top panels of Fig. 4) is expected to allow the determination of the $x_{B j}$ position of the node of this function, which is important for the global fitting procedure.

The GPD $E$, which is of great interest to investigate the Ji sum rule [17] which relates the total angular momentum carried by a quark or a gluon, can in principle be assessed using a transversely polarised target. Such a measurement is envisaged for a later stage of the COMPASS programme.

\section{References}

[1] S. Meissner, A. Metz, M. Schlegel, JHEP 0908, 056 (2009)

[2] COMPASS Collaboration, P. Abbon et al., Nucl. Instrum. Meth.A 577, 455 (2007)

[3] COMPASS Collaboration, F. Gautheron et al., CERN-SPSC-2010-014, SPSC-P-340, May 2010

[4] COMPASS Collaboration, C. Adolph et al., Phys. Lett. B 717, 376 (2012)

[5] HERMES Collaboration, A. Airapetian et al., Phys. Lett. B 693, 11 (2010)

[6] COMPASS Collaboration, E.S. Ageev et al., Nucl Phys. B 765, 31 (2007)

[7] COMPASS Collaboration, M. Alexkseev et al., Phys. Lett. B 673, 376 (2009)

[8] M. Anselmino et al., Phys. Rev. D 87, 094019 (2013)

[9] BELLE Collaboration, R. Seidl et al., Phys. Rev. D 86, 032011 (E) (2012)

[10] HERMES Collaboration, A. Airapetian et al., Phys. Rev. Lett. B 103, 152002 (2009)

[11] J.C. Collins, Phys. Lett. B 536, 43 (2002)

[12] M. Anselmino et al., Phys. Rev. D 79, (2009)

[13] A.V. Belitsky, D. Mueller, A. Kirchner, Nucl Phys. B 629, 323 (2002)

[14] M. Vanderhaeghen et al., Phys. Rev. D 60, 094017 (1999); K. Goeke et al., Prog. Part. Nucl. Phys.47, 401 (2001)

[15] A.V. Belitsky, D. Mueller, Nucl Phys. B 841, 1 (2010)

[16] HERMES Collaboration, A. Airapetian et al., JHEP 07, 032 (2012)

[17] X.-D. Ji, Phys. Rev. Lett. 78, (1997) 610; Phys. Rev. D 55, 7114 (1997) 